\title{
Neighborhood Environment Characteristics and Control of Hypertension and Diabetes in a Primary Care Patient Sample
}

\author{
M. Maya McDoom, PhD, MPH', ${ }^{1,2}$ Lisa A. Cooper, MD, MPH 1,2,3,4,5, Yea-Jen Hsu, PhD², \\ Abhay Singh, $B A^{3}$, Jamie Perin, $P h D^{2}$, and Rachel L. J. Thornton, $M D, P h D^{1,3,5}$ (D)
}

'Johns Hopkins Bloomberg School of Public Health, Baltimore, MD, USA; ${ }^{2}$ Welch Center for Prevention, Epidemiology and Clinical Research, Baltimore, MD, USA; ${ }^{3}$ Johns Hopkins School of Medicine, Baltimore, MD, USA; ${ }^{4}$ Johns Hopkins School of Nursing, Baltimore, MD, USA; ${ }^{5}$ Johns Hopkins Center for Health Equity, Baltimore, MD, USA.

BACKGROUND: Hypertension control and diabetes control are important for reducing cardiovascular disease burden. A growing body of research suggests an association between neighborhood environment and hypertension or diabetes control among patients engaged in clinical care.

OBJECTIVE: To investigate whether neighborhood conditions (i.e., healthy food availability, socioeconomic status (SES), and crime) were associated with hypertension and diabetes control.

DESIGN: Cross-sectional analyses using electronic medical record (EMR) data, U.S. Census data, and secondary data characterizing neighborhood food environments. Multivariate logistic regression analyses adjusted for potential confounders. Analyses were conducted in 2017.

PARTICIPANTS: Five thousand nine hundred seventy adults receiving primary care at three Baltimore City clinics in 2010-2011.

MAIN MEASURES: Census tract-level neighborhood healthy food availability, neighborhood SES, and neighborhood crime. Hypertension control defined as systolic blood pressure $<140 \mathrm{mmHg}$ and diastolic blood pressure $<90$ mmHg. Diabetes control defined as HgbAlc $<7$.

KEY RESULTS: Among patients with hypertension, neighborhood conditions were not associated with lower odds of blood pressure control after accounting for patient and physician characteristics. However, among patients with diabetes, in fully adjusted models accounting for patient and physician characteristics, we found that patients residing in neighborhoods with low and moderate SES had reduced odds of diabetes control $(\mathrm{OR}=0.74(95 \%$ $\mathrm{CI}=0.57-0.97)$ and $\mathrm{OR}=0.75$ (95\% CI = 0.57-0.98), respectively) compared to those living in high-SES neighborhoods.

CONCLUSIONS: Neighborhood disadvantage may contribute to poor diabetes control among patients in clinical care. Community-based chronic disease care management strategies to improve diabetes control may be

Electronic supplementary material The online version of this article (https://doi.org/10.1007/s11606-020-05671-y) contains supplementary material which is available to authorized users.

Prior Presentations An abstract of this article was presented at the AHA/EPI|Lifestyle Conference in New Orleans, LA, on March 20-23, 2018.

Received May 21, 2019

Accepted January 13, 2020

Published online February 10, 2020 optimally effective if they also address neighborhood SES among patients engaged in care.

J Gen Intern Med 35(4):1189-98

DOI: $10.1007 / \mathrm{s} 11606-020-05671-y$

(C) Society of General Internal Medicine 2020

\section{INTRODUCTION}

Hypertension control and diabetes control are important for reducing cardiovascular disease (CVD) morbidity and mortality ${ }^{1}$. Individuals with hypertension have a $63.4 \%$ lifetime risk of developing CVD compared to $46.1 \%$ among those with normal blood pressure ${ }^{2}$. Moreover, each 10-year increase in duration of diabetes is also associated with increased CVD risk and mortality ${ }^{3}$. Despite the availability and effectiveness of lifestyle and pharmacological interventions, many patients engaged in care do not achieve optimal blood pressure or diabetes control ${ }^{4,5}$. Patient, provider, and health system factors, such as poor medication adherence; limited healthcare access; and variation in diagnosis, treatment, and adherence to clinical guidelines are related to poor hypertension and diabetes control ${ }^{6,7}$.

Furthermore, racial/ethnic disparities persist despite improvement in overall hypertension and diabetes control ${ }^{5,8}$. Data from the National Health and Nutrition Examination Survey (NHANES) estimate that, among hypertensive adults in the USA, non-Hispanic Whites achieved hypertension control at higher rates (56\%) as compared to non-Hispanic Blacks (48\%) and Hispanics $(47 \%)^{8}$. NHANES data suggest similar disparities in disease control among adults in the USA with diabetes. For example, among adults in the USA with diabetes, $52 \%$ of non-Hispanic Blacks and $43 \%$ of Mexican Americans achieved glycemic control versus $57 \%$ of non-Hispanic Whites ${ }^{5}$. Racial/ ethnic disparities in diabetes and hypertension control are major public health issues in their own right, and they likely also contribute to disproportionately increased risk of CVD complications, including kidney disease and strokes among nonHispanic Blacks and Mexican Americans ${ }^{9,10}$.

Most research has focused on patient-level and health system factors, but, increasingly, research has examined whether 
neighborhood factors influence hypertension and diabetes control among clinical populations. In prior research, neighborhood characteristics including neighborhood socioeconomic status (SES) ${ }^{11}$, healthy food availability, pollution, crime, and walkability have been associated with $\mathrm{CVD}^{12}$. Disadvantaged neighborhood conditions may undermine diabetes and hypertension control by inhibiting individuals' ability to adopt healthy lifestyle behaviors, generating chronic stress, and producing physiological changes increasing CVD risk $^{12}$. Previous work examining the relationship between the neighborhood food environment and diabetes or hypertension has used supermarket presence, the number of fast-food restaurants, and the number of food outlets ${ }^{13}$.

This study builds on prior research by using food environment data derived from direct observations of every food store in Baltimore, MD, using a standardized audit tool, the Healthy Food Availability Index (HFAI). The HFAI generates a numeric score for every food store in Baltimore City that can be aggregated to the census tract level to produce an overall measure of the neighborhood healthy food availability ${ }^{14}$. Previous work using the HFAI has found that $43 \%$ of predominantly Black neighborhoods in Baltimore City are in the lowest tertial of health food availability versus $4 \%$ of predominately white neighborhoods ${ }^{15}$. Furthermore, low availability of healthy food has been associated with high diabetes prevalence ${ }^{16}$, hypertension prevalence ${ }^{17}$, and a lower-quality diet ${ }^{15}$, which could negatively impact diabetes control.

This study extends prior research by examining relationships between distinct features of the neighborhood food environment and social environment (i.e., healthy food availability and crime, respectively) in addition to traditional measures of neighborhood SES, with hypertension and diabetes control among patients in primary care. As such, it adds to a small but growing body of evidence exploring the association of neighborhood conditions and the odds of diabetes and hypertension control among an adult population in the USA who are engaged in care. We hypothesized that neighborhood environments with high SES, healthy food availability and low crime would be associated with greater odds of hypertension and diabetes control.

\section{METHODS}

\section{Study Population}

The study population is adults aged $\geq 18$ years with hypertension and/or diabetes who received primary care at three practices in Baltimore, MD, belonging to a network of more than 35 clinics across Maryland. The clinics share an electronic medical record (EMR) system and participated in a multilevel system quality improvement intervention study designed to reduce disparities in blood pressure control - details are described elsewhere ${ }^{18}$.
The present study is cross-sectional. We used baseline data prior to intervention implementation in each clinic to assess clinical outcomes of diabetes and hypertension control in this study. This approach was used to avoid confounding from the effects of the quality improvement intervention on blood pressure outcomes. The analytic sample included all patients who received care at the three clinics in Baltimore, who had complete address information and resided within the city limits. Patient's addresses were geocoded and assigned to a census tract, which was the geographic unit of analysis used to define a neighborhood for this study. Patients' mailing addresses listed in the EMR were used to assign neighborhood of residence and were geocoded to census tracts using ArcGIS software, version 10.2 (Environmental Systems Research Institute, Inc., Redlands, California). Because the study focused on examining the association of neighborhood conditions and diabetes and hypertension control among patients who were engaged in care, this analysis excluded patients if they did not have at least one clinical encounter between October 2010 and September 2011 prior to the start of the quality improvement intervention, did not have a diagnosis of hypertension or diabetes, or did not have a blood pressure or $\mathrm{HgbAl}$ c measure within 2 years before the intervention start date at a given site (Fig. 2 in the Appendix). The final analytic sample included individuals who were either Black or White and had complete data for all variables retained $(n=5711)$. The final sample was restricted to only those patients who were identified in the EMR as Black or White because there were insufficient sample sizes for patients of other racial/ethnic groups for subgroup analyses. The Johns Hopkins Medicine Institutional Review Board approved the study. Informed consent was waived for patients. Providers implied consent by agreeing to complete the survey.

\section{Measures}

The exposures were neighborhood SES, crime, and healthy food availability. Neighborhood SES was characterized using a method described elsewhere by generating a summary score of six z-scores for domains measured at the census tract level. Given the timing of the clinical outcome data from the EMR that was used in this study, we used data from the 2010 U.S. Census to characterize neighborhood SES. The 6-domain summary score used in this analysis has been used in other research studies to characterize neighborhood SES ${ }^{19}$. The six domains included in the neighborhood SES summary score were median household income; median housing value, percent of owneroccupied households; proportion of adults $>25$ years with a high school education; proportion of adults $>25$ years with a college education; and proportion of adults $>16$ years with executive, managerial, or professional occupations ${ }^{19}$. A higher summary score represents a less-deprived neighborhood environment. Neighborhood SES tertiles were categorized as low ( -7.01 to -0.66$)$, medium ( -0.54 to 1.13$)$, and high (1.21 to 4.76). Prior research suggests that while neighborhood SES is correlated with individual SES, it represents a distinct construct 
such that there is heterogeneity in individual-level SES among residents in the same neighborhoods ${ }^{19}$. Furthermore, neighborhood disadvantage is a separate source of disadvantage from individual SES and may represent a distinct exposure as compared to individual SES ${ }^{20}$.

Neighborhood crime was characterized using data from the Baltimore City Police Department between 2006 and $2010^{21}$. Neighborhood crime was defined as a count of violent crimes per square mile at the census tract level and included offenses involving force or threat of force such as homicide/manslaughter, rape, aggravated assault, and robbery. Crime tertiles were categorized as low (29.88 to 437.60 ), medium (443.04 to 1395.99), and high (1407.77 to 4474.05).

The Baltimore HFAI was used to characterize neighborhood healthy food availability. The HFAI has been validated by previous work and is described elsewhere ${ }^{14}$. Briefly, the HFAI assessed the following: 100\% fruit juice; fresh fruit and vegetables; meats; chicken; seafood; frozen fruits, vegetables and healthy meals; rice, beans, and pasta; canned fruits and vegetables; $100 \%$ whole wheat and other bread; and low-sugar cereal ( $7 \mathrm{~g}$ of sugar or less). This study utilizes the version of the HFAI data collected in 2012, which was collected by auditing 900 stores in Baltimore, MD, using a streamlined tool. Data on the food available in each store is aggregated to the geographic unit of interest to characterize the healthy food availability in a given neighborhood. In this study, the neighborhood is defined at the census tract level. Therefore, HFAI ratings for every store within a given census tract are combined to yield a census trace summary HFAI score. These scores ranged from 0 to 28.5 points, with a higher score indicating a greater availability of healthy foods. HFAI tertiles were categorized as low ( 0.00 to 7.50$)$, medium (7.55 to 10.17), and high (10.38 to 28.5$)$. Lower HFAI tertiles were found to be correlated with lower-quality diets ${ }^{15}$.

Hypertension control and diabetes control were the primary outcomes and assessed at the most recent clinical visit before each clinic's intervention start date. Hypertension control was defined as systolic blood pressure $<140 \mathrm{mmHg}$ and diastolic blood pressure $<90 \mathrm{mmHg}$ according to criteria from the Seventh Joint National Committee on Hypertension ${ }^{22}$. Diabetes control was defined as HgbAlc $<7$ based on the American Diabetes Association recommendations ${ }^{23}$. Both hypertension control and diabetes control were binary variables $(1=$ controlled, $0=$ uncontrolled).

Age, gender, race (Black, White), marital status (married, not married), and insurance status (Medicaid, Medicare, private, uninsured, and unknown) were obtained from the EMR. Data on patients' individual or family income was not available from the EMR, nor was data on patient education level. Thus, insurance type was considered as a proxy for individual SES, insofar as Medicaid insurance, in particular, is a meanstested program intended for low-income patients. Among the Medicare population, reliable data was not available from the EMR to distinguish those patients who were dually eligible for Medicare and Medicaid (i.e., low-income older adults) from other Medicare beneficiaries who were not low-income. Variables partially mediating or cofounding the association between neighborhood environment and hypertension or diabetes control such as biomedical risk factors (e.g., body mass index, comorbidities) and health behaviors (e.g., smoking) were also obtained from the EMR. Body mass index (BMI) was calculated by dividing measured weight in kilograms by height in meters squared and further categorized as underweight, normal weight, overweight, and obese. Comorbidities were assessed using the Charlson Comorbidity Index, which takes into account the number and severity of comorbid diseases ${ }^{24}$. Smoking was assessed and reported in the EMR and was categorized as current, former, or never smoker.

\section{Statistical Analysis}

We examined patient characteristics using means and standard deviations or medians with interquartile range for continuous variables, and frequencies and percentages for categorical variables. $t$ tests were used to compare continuous variables and chi-square tests to compare categorical variables across patients with and without hypertension and diabetes control.

Multivariate logistic regression models were constructed to examine associations between neighborhood environment characteristics and hypertension control (Table 2) and diabetes control (Table 3). To account for within census tract correlation, all models included clustering at census tract through robust estimation of standard errors using a clustered sandwich estimator. Because neighborhood environment characteristics are likely to be correlated, we evaluated the association of neighborhood conditions with diabetes and hypertension control using separate models for each neighborhood exposure. We used logistic regression to separately estimate the associations of neighborhood characteristics on disease control, sequentially adjusting for patient demographic characteristics in model 2 (i.e., age, race, and gender), and other patient factors that were considered as correlates or confounders were added to model 3 . These included patient biomedical and health behavior risk factors (i.e., BMI, smoking status, Charlson Comorbidity Index), marital status, and insurance type.

In sensitivity analyses, we further adjusted for provider characteristics (i.e., the number of years for each provider in practice since completing training, position/specialty, and provider gender) as these factors may influence the association between neighborhood environment characteristics and hypertension or diabetes control. These are reflected in model 4 . We were unable to adjust for provider race because provider data were collected from a publicly available source not reporting race or ethnicity. Additional sensitivity analyses tested for effect modification by patient race and neighborhood exposure because some prior research suggests that diabetes and hypertension control may be associated with patient race ${ }^{25}$.

Stata (version 14.0; StataCorp LP, College Station, Texas) was used to perform all statistical analyses in 2017. Statistical significance was defined a priori as a 2 -sided $p$ value $<0.05$. 


\section{RESULTS}

The sample included patients residing in 197 of 200 census tracts in Baltimore, MD. Patients' missing data represented a small proportion (4\%) of the eligible sample. Analyses were conducted on two non-mutually exclusive samples that were derived from the final analytic sample and represented those with hypertension $(n=5325)$ or diabetes $(n=2094)$. Table 1 compares patients with controlled versus uncontrolled hypertension or diabetes with respect to patient demographic characteristics, risk factors, comorbidities, marital status, and insurance type. In our study of patients engaged in clinical care at three Baltimore City clinics, patients with either controlled hypertension or diabetes were more likely to be White, older, former smokers, and normal or overweight compared to those who were uncontrolled. Individuals with controlled hypertension were more likely to have more comorbidities compared to those who were not controlled (Table 1). Those with controlled diabetes were more likely to be female or covered by Medicare than those with uncontrolled diabetes. When examining neighborhood characteristics by race, White patients resided in more advantaged neighborhood environments (higher neighborhood SES, high availability of healthy foods, low crime) compared to Black patients (Fig. 1). Study patients' primary care providers completed training on average $20.3 \pm 9.9$ years ago; most were female $(n=3775 ; 86.6 \%)$ and were internal medicine physicians $(n=3699 ; 84.9 \%)$.] $\rightarrow$

Among those with hypertension, residing in low or medium neighborhood SES tertiles was associated with a decreased odds of hypertension control; however, this association was attenuated in the fully adjusted model (Table 2). Being in a high-crime neighborhood was not statistically significantly associated with decreased odds of hypertension control, nor was there a statistically significant association of HFAI and hypertension control. Being Black, a current smoker, and having Medicaid insurance or being uninsured were associated with statistically significantly decreased odds of hypertension control in adjusted models (Table 2, model 2 and model 3 , respectively) examining the association of neighborhood SES, neighborhood crime, and neighborhood healthy food availability with hypertension control. Adjusting for physician characteristics (Table 2, model 4) did not attenuate the association of patient race or being uninsured with odds of blood pressure control, but the association of Medicaid insurance with lower odds of blood pressure control was no longer statistically significant. Of the physician characteristics added to model 4, only provider's level of experience was statistically significant and was positively associated with odds of blood pressure control in models examining the association of neighborhood SES, neighborhood healthy food availability, and neighborhood crime with blood pressure control.

Among those with diabetes, living in low- and moderate-SES neighborhoods, in low HFAI neighborhoods, or in high-crime neighborhoods was associated with a decreased odds of diabetes control, yet not statistically significant in unadjusted results. These associations remained relatively weak and not statistically significant even after adjustment for covariates (Table 3).

Table 1 Descriptive Characteristics of 5970 Primary Care Patients Diagnosed with Diabetes (2094, 36\%) or Hypertension (5325, 89\%)

\begin{tabular}{|c|c|c|c|c|c|c|c|}
\hline & \multirow{3}{*}{$\begin{array}{l}\text { Overall } \\
N=\mathbf{5 9 7 0}\end{array}$} & \multicolumn{3}{|c|}{ Diabetes control } & \multicolumn{3}{|c|}{ Hypertension control } \\
\hline & & \multirow{2}{*}{$\frac{\text { No }}{N=1024}$} & \multirow{2}{*}{$\frac{\text { Yes }}{N=1070}$} & \multirow{2}{*}{$\begin{array}{l}p \\
\text { value }\end{array}$} & \multirow{2}{*}{$\frac{N o}{N=2214}$} & \multirow{2}{*}{$\frac{\text { Yes }}{N=3111}$} & \multirow[t]{2}{*}{$p$ value } \\
\hline & & & & & & & \\
\hline \multicolumn{8}{|l|}{ Gender, $n(\%)$} \\
\hline Female & $3988(66.8)$ & $650(63.5)$ & $742(69.3)$ & 0.004 & $1475(66.6)$ & $2107(67.7)$ & 0.40 \\
\hline \multicolumn{8}{|l|}{ Race, $n(\%)$} \\
\hline Black, non-Hispanic & $5221(87.8)$ & $954(93.2)$ & $969(90.6)$ & 0.03 & $2050(92.6)$ & $2731(87.8)$ & $<0.001$ \\
\hline Age in 2011, median (IQR) & $\begin{array}{l}57.7(48.5, \\
67.8)\end{array}$ & $\begin{array}{l}57.5(49.2, \\
66.1)\end{array}$ & $\begin{array}{l}62.3(54.0, \\
73.0)\end{array}$ & $<0.001$ & $\begin{array}{l}57.2(48.3, \\
67.6)\end{array}$ & $\begin{array}{l}59.1(50.0, \\
68.8)\end{array}$ & $<0.001$ \\
\hline \multicolumn{8}{|l|}{ Smoking, $n(\%)$} \\
\hline Current & $1470(24.6)$ & $219(21.4)$ & $225(21.0)$ & 0.67 & $621(28.0)$ & $690(22.2)$ & $<0.001$ \\
\hline Former & $1788(30.0)$ & 327 (31.9) & $361(33.7)$ & & $579(26.2)$ & $1036(33.3)$ & \\
\hline \multirow{2}{*}{\multicolumn{8}{|c|}{ BMI in $\mathrm{kg} / \mathrm{m}^{2}, n(\%)$}} \\
\hline & & & & & & & \\
\hline Underweight $(<18.5)$ & $63(1.1)$ & $6(0.6)$ & $9(0.8)$ & $<0.001$ & $17(0.8)$ & $43(1.4)$ & $<0.001$ \\
\hline Normal weight (18.5-24.9) & $785(13.4)$ & $78(7.6)$ & $136(12.7)$ & & $287(13.0)$ & $419(13.5)$ & \\
\hline Overweight $(25.0-29.9)$ & $1491(25.5)$ & $210(20.5)$ & $260(24.3)$ & & $507(22.9)$ & $852(27.4)$ & \\
\hline Obese $(30.0+)$ & $3518(60.1)$ & $730(71.3)$ & $665(62.1)$ & & $1403(63.4)$ & $1797(57.8)$ & \\
\hline \multicolumn{8}{|l|}{ Insurance, $n(\%)$} \\
\hline Medicaid & $1061(17.8)$ & $182(17.8)$ & $152(14.2)$ & $<0.001$ & $440(19.9)$ & $483(15.5)$ & $<0.001$ \\
\hline Medicare & $1901(31.8)$ & 344 (33.6) & $453(42.3)$ & & $716(32.3)$ & $1031(33.1)$ & \\
\hline Private & $2790(46.7)$ & 457 (44.6) & $446(41.7)$ & & $967(43.7)$ & $1524(49.0)$ & \\
\hline Uninsured & $151(2.5)$ & $33(3.2)$ & $9(0.8)$ & & $62(2.8)$ & $47(1.5)$ & \\
\hline Unknown & $67(1.1)$ & $8(0.8)$ & $10(0.9)$ & & $29(1.3)$ & $26(0.8)$ & \\
\hline \multicolumn{8}{|l|}{ Marital status, $n(\%)$} \\
\hline Not married & $3959(66.3)$ & $662(64.6)$ & $698(65.2)$ & 0.78 & $1492(67.4)$ & $2027(65.2)$ & 0.09 \\
\hline $\begin{array}{l}\text { Charlson Comorbidity Index, mean } \\
\pm \mathrm{SD}\end{array}$ & $1.3 \pm 1.5$ & $2.1 \pm 1.6$ & $2.2 \pm 1.5$ & 0.12 & $1.2 \pm 1.5$ & $1.3 \pm 1.5$ & 0.032 \\
\hline
\end{tabular}

IQR interquartile range 
a

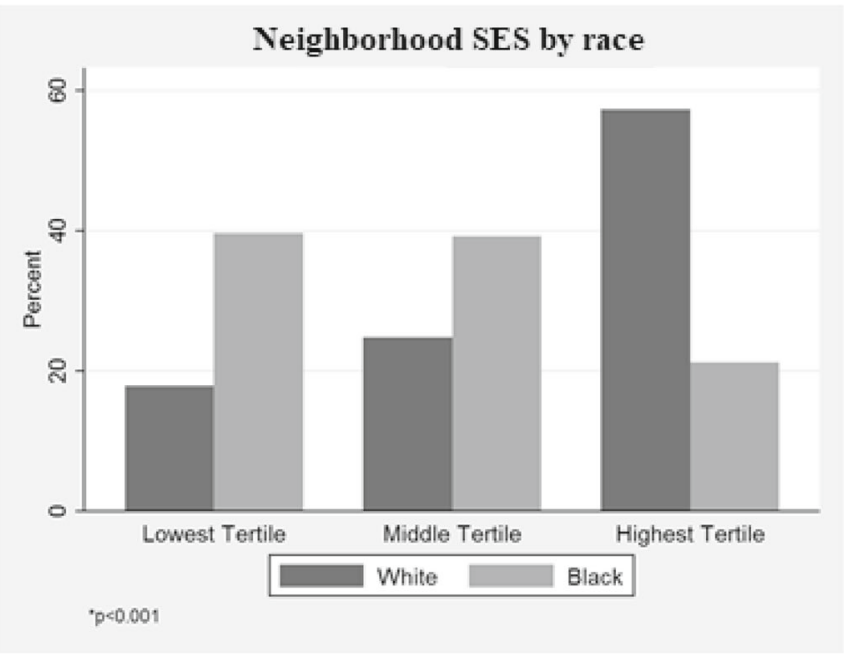

b

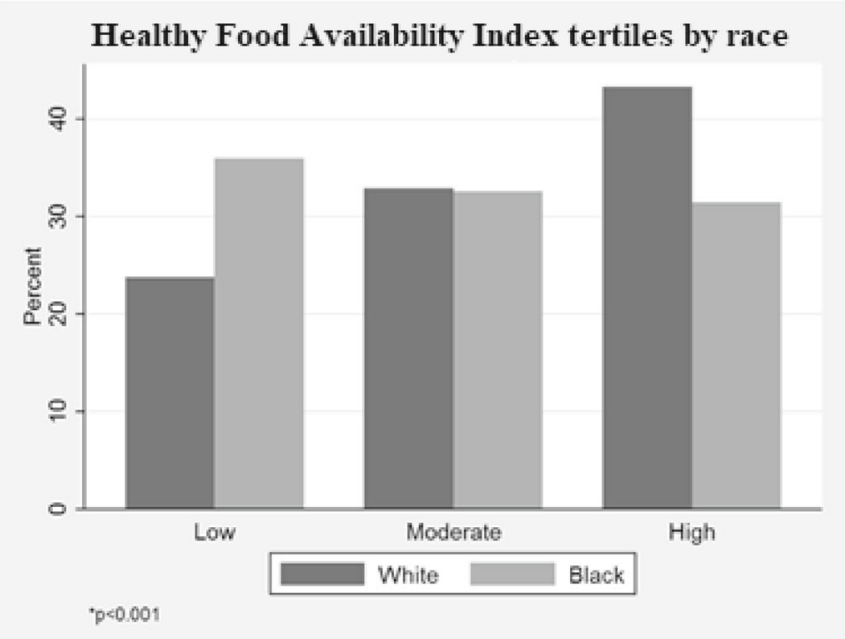

C

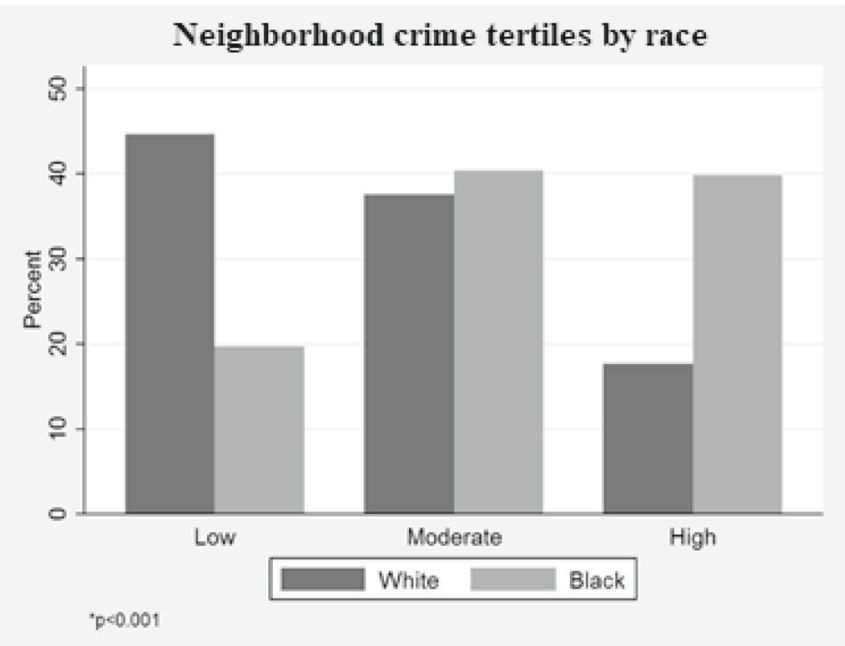

Fig. 1 a-c Distribution of neighborhood environment characteristics by race.

In additional sensitivity analyses (not shown), there were no statistically significant interactions between race and neighborhood environment characteristics in fully adjusted models for hypertension and diabetes control. Similar to the 
Table 2 Association Between Neighborhood Factors and Hypertension Control Among 5325 Primary Care Patients

\begin{tabular}{|c|c|c|c|c|}
\hline & Model 1* & Model $2^{\dagger}$ & Model 3 & Model $4^{\S}$ \\
\hline & OR (95\% CI) & OR $(95 \% \mathrm{CI})$ & OR (95\% CI) & OR $(95 \% \mathrm{CI})$ \\
\hline \multicolumn{5}{|c|}{ Neighborhood socioeconomic status } \\
\hline Low & $0.83(0.72-0.96)^{\mathrm{TI}}$ & $0.92(0.79-1.06)$ & $0.96(0.82-1.11)$ & $0.91(0.77-1.09)$ \\
\hline Medium & $0.85(0.74-0.98)^{\mathbb{\Psi}}$ & $0.93(0.80-1.07)$ & $0.94(0.81-1.08)$ & $0.88(0.75-1.04)$ \\
\hline High & Reference & Reference & Reference & Reference \\
\hline \multicolumn{5}{|l|}{ Race } \\
\hline Black & - & $0.60(0.49-0.73) * *$ & $0.64(0.53-0.79)^{* *}$ & $0.64(0.50-0.81)^{\sharp}$ \\
\hline White & - & Reference & Reference & Reference \\
\hline \multicolumn{5}{|l|}{ Insurance } \\
\hline Medicaid & & & $0.76(0.646-0.90)^{\mathrm{I}}$ & $0.88(0.72-1.07)$ \\
\hline Medicare & & & $0.87(0.758-1.00)$ & $0.90(0.77-1.06)$ \\
\hline Private & & & Reference & Reference \\
\hline Uninsured & & & $0.53(0.357-0.78)^{\#}$ & $0.56(0.35-0.90)^{\mathrm{q}}$ \\
\hline Unknown & & & $0.63(0.369-1.08)$ & $0.77(0.42-1.44)$ \\
\hline PCP years in practice" & & & & $1.01(1.01-1.02)^{\#}$ \\
\hline \multicolumn{5}{|c|}{ Healthy food availability index } \\
\hline Low & $0.99(0.85-1.14)$ & $1.03(0.89-1.20)$ & $1.01(0.89-1.16)$ & $0.97(0.83-1.14)$ \\
\hline Medium & $0.90(0.79-1.02)$ & $0.93(0.82-1.06)$ & $0.94(0.82-1.08)$ & $0.91(0.78-1.07)$ \\
\hline High & Reference & Reference & Reference & Reference \\
\hline \multicolumn{5}{|l|}{ Race } \\
\hline Black & - & $0.58(0.48-0.71)^{* *}$ & $0.63(0.52-0.77)^{* *}$ & $0.62(0.49-0.78)^{\#}$ \\
\hline White & - & Reference & Reference & Reference \\
\hline \multicolumn{5}{|l|}{ Insurance } \\
\hline Medicaid & & & $0.76(0.642-0.89)^{\#}$ & $0.87(0.71-1.06)$ \\
\hline Medicare & & & $0.87(0.753-0.99)^{\mathrm{I}}$ & $0.89(0.76-1.05)$ \\
\hline Private & & & Reference & Reference \\
\hline Uninsured & & & $0.53(0.355-0.78)^{\#}$ & $0.56(0.35-0.91)^{\mathrm{q}}$ \\
\hline Unknown & & & $0.63(0.368-1.08)$ & $0.77(0.42-1.44)$ \\
\hline PCP years in practice" & & & & $1.02(1.01-1.03)^{\#}$ \\
\hline \multicolumn{5}{|l|}{ Neighborhood crime } \\
\hline Medium & $0.87(0.75-1.01)$ & $0.92(0.79-1.07)$ & $0.93(0.81-1.09)$ & $0.92(0.78-1.09)$ \\
\hline High & $0.82(0.70-0.95)^{* *}$ & $0.89(0.76-1.03)$ & $0.94(0.81-1.10)$ & $1.00(0.84-1.19)$ \\
\hline Low & Reference & Reference & Reference & Reference \\
\hline \multicolumn{5}{|l|}{ Race } \\
\hline Black & - & $0.60(0.49-0.73) * *$ & $0.64(0.52-0.78) * *$ & $0.62(0.49-0.78)^{\#}$ \\
\hline White & - & Reference & Reference & Reference \\
\hline \multicolumn{5}{|l|}{ Insurance } \\
\hline Medicaid & & & $0.76(0.647-0.90)^{\#}$ & $0.87(0.71-1.06)$ \\
\hline Medicare & & & $0.87(0.759-1.00)$ & $0.89(0.76-1.05)$ \\
\hline Private & & & Reference & Reference \\
\hline Uninsured & & & $0.53(0.359-0.79)^{\#}$ & $0.57(0.35-0.91)^{\mathrm{q}}$ \\
\hline Unknown & & & $0.63(0.369-1.09)$ & $0.77(0.41-1.44)$ \\
\hline PCP years in practice"l & & & & $1.02(1.01-1.03)^{\#}$ \\
\hline
\end{tabular}

Values in italics indicate statistical significance $\left(" p<0.05,{ }^{*} p<0.01, * * p<0.001\right)$

*Model 1: neighborhood factor

${ }^{\dagger}$ Model 2: model $1+$ race + age + gender

${ }^{*}$ Model 3: model $2+$ married + BMI + smoking status + insurance + Charlson Comorbidity Index

${ }_{1}$ Model 4: model $3+$ years PCP trained + PCP position + PCP gender

"Number of years in practice since training was completed

main results, neighborhood environment factors were not significantly related to hypertension control in adjusted multivariate models. However, there was a $26 \%$ and $25 \%$ decreased odds for diabetes control among individuals living in low-SES $(\mathrm{OR}=0.74 ; 95 \% \mathrm{CI}=0.57-0.97)$ or moderate-SES $(\mathrm{OR}=$ $0.75 ; 95 \% \mathrm{CI}=0.57-0.98)$ neighborhood (Table 3 ).

\section{DISCUSSION}

In this large clinic-based study of neighborhood environment conditions and hypertension and diabetes control among patients engaged in care, we did not find consistent and independent associations of neighborhood SES, neighborhood healthy food availability, or neighborhood crime with hypertension control. We did, however, find statistically significant associations of low- and moderateSES neighborhoods versus high-SES neighborhoods and lower odds of diabetes control in models adjusted for patient and provider level factors. As such, these findings are an important addition to the growing literature examining the relationship of neighborhood factors with achieving hypertension and diabetes control among patients engaged in care and draw patients from across the vast majority of neighborhoods in Baltimore, MD.

In addition to findings suggesting lower odds of diabetes control for patients living in low- or moderate-SES 
Table 3 Association Between Neighborhood Factors and Diabetes Control Among 2094 Primary Care Patients

\begin{tabular}{|c|c|c|c|c|}
\hline & Model 1* & Model $2^{\dagger}$ & Model 3* & Model $4^{\S}$ \\
\hline & OR $(95 \% \mathrm{CI})$ & OR $(95 \% \mathrm{CI})$ & OR $(95 \% \mathrm{CI})$ & OR $(95 \%$ CI $)$ \\
\hline \multicolumn{5}{|c|}{ Neighborhood socioeconomic status } \\
\hline Low & $0.82(0.65-1.02)$ & $0.86(0.69-1.08)$ & $0.85(0.68-1.09)$ & $0.74(0.57-0.97)^{q}$ \\
\hline Medium & $0.86(0.69-1.08)$ & $0.85(0.67-1.08)$ & $0.87(0.68-1.10)$ & $0.75(0.57-0.98)^{\mathrm{q}}$ \\
\hline High & Reference & Reference & Reference & Reference \\
\hline \multicolumn{5}{|l|}{ Race } \\
\hline Black & - & $0.77(0.55-1.08)$ & $0.79(0.57-1.11)$ & $0.78(0.53-1.14)$ \\
\hline White & - & Reference & Reference & Reference \\
\hline \multicolumn{5}{|l|}{ Insurance } \\
\hline Medicaid & & & $1.03(0.778-1.35)$ & $1.08(0.77-1.50)$ \\
\hline Medicare & & & $0.99(0.797-1.22)$ & $0.96(0.75-1.22)$ \\
\hline Private & & & Reference & Reference \\
\hline Uninsured & & & $0.34(0.161-0.73)^{\#}$ & $0.43(0.18-1.03)$ \\
\hline Unknown & & & $1.52(0.588-3.94)$ & $1.71(0.58-5.08)$ \\
\hline PCP years in practice ${ }^{\|}$ & & & & $1.00(0.99-1.01)$ \\
\hline \multicolumn{5}{|c|}{ Healthy food availability index } \\
\hline Low & $0.90(0.72-1.13)$ & $0.92(0.73-1.17)$ & $0.98(0.79-1.22)$ & $0.95(0.74-1.22)$ \\
\hline Medium & $0.97(0.79-1.18)$ & $0.96(0.78-1.18)$ & $0.91(0.73-1.13)$ & $0.89(0.69-1.14)$ \\
\hline High & Reference & Reference & Reference & Reference \\
\hline \multicolumn{5}{|l|}{ Race } \\
\hline Black & - & $0.74(0.54-1.03)$ & $0.76(0.55-1.07)$ & $0.73(0.50-1.06)$ \\
\hline White & - & Reference & Reference & Reference \\
\hline \multicolumn{5}{|l|}{ Insurance } \\
\hline Medicaid & & & $1.02(0.769-1.34)$ & $1.05(0.76-1.47)$ \\
\hline Medicare & & & $0.98(0.786-1.21)$ & $0.93(0.73-1.19)$ \\
\hline Private & & & Reference & Reference \\
\hline Uninsured & & & $0.34(0.157-0.71)^{\#}$ & $0.41(0.17-0.99)^{\mathrm{q}}$ \\
\hline Unknown & & & $1.50(0.579-3.88)$ & $1.62(0.54-4.80)$ \\
\hline PCP years in practice & & & & $1.00(0.98-1.01)$ \\
\hline \multicolumn{5}{|l|}{ Crime } \\
\hline Medium & $0.94(0.74-1.19)$ & $0.97(0.76-1.24)$ & $0.99(0.78-1.27)$ & $1.02(0.77-1.35)$ \\
\hline High & $0.76(0.61-0.96)^{\mathrm{T}}$ & $0.81(0.64-1.04)$ & $0.81(0.63-1.04)$ & $0.86(0.65-1.14)$ \\
\hline Low & Reference & Reference & Reference & Reference \\
\hline \multicolumn{5}{|l|}{ Race } \\
\hline Black & - & $0.77(0.55-1.08)$ & $0.79(0.56-1.11)$ & $0.74(0.51-1.08)$ \\
\hline White & - & Reference & Reference & Reference \\
\hline \multicolumn{5}{|l|}{ Insurance } \\
\hline Medicaid & & & $1.04(0.791-1.38)$ & $1.07(0.79-1.49)$ \\
\hline Medicare & & & $1.01(0.809-1.24)$ & $0.95(0.74-1.22)$ \\
\hline Private & & & Reference & Reference \\
\hline Uninsured & & & $0.36(0.166-0.76)^{\#}$ & $0.42(0.17-1.03)$ \\
\hline Unknown & & & $1.56(0.602-4.05)$ & $1.63(0.55-4.83)$ \\
\hline PCP years in practice & & & & $0.99(0.98-1.01)$ \\
\hline
\end{tabular}

Values in italics indicate statistical significance $\left(" p<0.05,{ }^{*} p<0.01, * * p<0.001\right)$

*Model 1: neighborhood factor

${ }^{\dagger}$ Model 2: model 1 + race + age + gender

${ }^{*}$ Model 3: model $2+$ married + BMI + smoking status + insurance + Charlson Comorbidity Index

${ }^{S}$ Model 4: model $3+$ years $P C P$ trained $+P C P$ position + PCP gender

"Number of years in practice since training was complete

versus high-SES neighborhoods, we also found that, even when holding neighborhood conditions constant, being Black and having Medicaid insurance or being uninsured were associated with lower odds of hypertension control even when adjusting for other patient demographic characteristics, biomedical risk factors, health behaviors, and chronic disease burden. Furthermore, only the association of Medicaid insurance with lower odds of hypertension control was attenuated by adjusting for physician characteristics. Similarly, being uninsured was associated with lower odds of diabetes control even when holding neighborhood conditions constant and adjusting for patient demographic characteristics, biomedical risk factors, health behaviors, and chronic disease burden.
Findings from this study suggest that disparities in hypertension and diabetes control cannot be explained entirely based on differences in neighborhood conditions. In fact, our sample patient race and insurance type were the factors most consistently associated with odds of hypertension and diabetes control. In this way, our findings differ from prior studies examining the associations of neighborhood factors with chronic conditions insofar that this research does not detect a main effect of neighborhood conditions on hypertension control in any of the adjusted models. In contrast, a previous population-based investigation found residents who lived continuously in the most advantaged residential areas achieved glycemic control in a shorter time compared to those in disadvantaged areas 
(9.9 months vs. 11.5 months) ${ }^{26}$. Moreover, in a randomized controlled trial to improve medication adherence among diabetic patients in Pennsylvania primary care clinics, investigators found patients residing in advantaged neighborhoods with high social affluence and residential stability were more likely to be adherent to medication and ultimately achieve glycemic control ${ }^{27}$.

Black patients in our study were less likely to achieve hypertension control compared to Whites. This observation is consistent with population-based or communitybased study findings ${ }^{5,28}$. Even though we found Blacks had decreased odds of control, neighborhood impact upon health is often inadequately discussed and seldom incorporated into treatment plans ${ }^{29}$. However, it is an oversimplification to suggest that race is a more important determinant of blood pressure control than neighborhoods. Our findings highlight key areas where further research is needed and raise important considerations about the pros and cons of leveraging clinical administrative datasets from EMRs to investigate the association of neighborhood conditions and clinical outcomes such as hypertension and diabetes control.

Several mechanisms may explain our findings. While healthcare access is a key component to achieve hypertension and diabetes control, patients living in disadvantaged neighborhoods remain susceptible to the adverse conditions of these environments. This highlights the interconnectedness of individual characteristics and social context and the difficulty in fully disentangling, describing, and understanding the independent associations of individual and structural factors on disease morbidity and chronic disease management. For example, neighborhood socioeconomic environment can influence the availability of resources promoting hypertension and diabetes control, such as grocery stores ${ }^{30}$ and recreational facilities which may influence diet and physical activity ${ }^{12}$. Although these findings have been well established in population-based studies of disease prevalence, our results examining the association of neighborhood conditions with diabetes and hypertension control were mixed. Residents living in a disadvantaged neighborhood might also have increased exposure to crime. Exposure to high crime is related to perceived lack of safety which may contribute to lack of engagement in healthy behaviors important for control, such as physical activity ${ }^{31}$. Moreover, crime is a neighborhood environmental stressor that may trigger biological pathways which modulate physiologic responses to stress ${ }^{12}$.

Independent of primary care provider characteristics, individuals living in economically deprived neighborhoods were less likely to achieve diabetes control in our study, suggesting while providers are important for clinical management, neighborhood determinants are likely instrumental in diabetes self-care, such as adherence to medication, clinical visits, and uptake of lifestyle changes
${ }^{32}$. Furthermore, the comparability of neighborhoods may need to consider other aspects of the neighborhood environment beyond neighborhood SES, food environment, or crime indicators per se. For example, it may be difficult to disentangle neighborhood characteristics and racial identity in primary care settings insofar as Black patients embody the social and material conditions in which they live ${ }^{33}$ and the historical context in which racial segregation developed in Baltimore. For example, systematic implementation of redlining policies in Baltimore not only contributed to racial segregation but also produced socioeconomic neighborhood conditions and health inequalities affecting Blacks and Whites differently ${ }^{34,}{ }^{35}$. This, in turn, can affect where patients access and engage in care, how providers approach the care of patients living in deprived neighborhoods, and ultimately, whether patients achieve better health outcomes ${ }^{36}$.

The 2019 ACC/AHA Guideline on the Primary Prevention of Cardiovascular Diseases includes the following take-home message: "A team-based care approach is an effective strategy for the prevention of cardiovascular disease. Clinicians should evaluate the social determinants of health that affect individuals to inform treatment decisions." ${ }^{37}$ However, a recent report from the National Academies of Sciences, Engineering, and Medicine highlights that contextual risks to health associated with living in disadvantaged neighborhoods are not overcome by engaging in care as it is currently delivered in most settings across the USA ${ }^{38}$. The report suggests a robust framework is needed to insure healthcare settings are optimally equipped to integrate social care via five types of activities: awareness, adjustment, assistance, alignment, and advocacy. Clinicians and other members of the care team can increase efforts to elicit information about neighborhood conditions and other social determinants during medical encounters ${ }^{29}$. Standardized screening tools can improve data gathering in this regard ${ }^{39}$. Screening for social determinants could occur with the intent to adjust clinical decision-making to be more responsive to patients' social situations (e.g., less expensive medications or reduced insulin dosages at the end of the month if patients' food benefits run out and they have to eat less). Clinicians could also use this information to assist patients, linking them to community or population management programs that support identified needs. Examples include activating case managers or community health workers to assist patients with self-management support ${ }^{32}$ and navigating external bureaucracies or daily life challenges such as obtaining transportation or food vouchers and even extending the reach of the clinic through home visits. An alignment or advocacy strategy might be for the health system to engage in a medical legal partnership that addresses common legal needs of families such as housing conditions or personal safety ${ }^{34,35}$. 


\section{Limitations and Strengths}

Our study has several limitations. First, the EMR has incomplete information about individual-level SES variables, such as income or education; thus, we were limited to identifying individual SES through insurance status as a proxy. Additionally, we used cross-sectional data unable to account for timevariant measures or inferences about causality; thus, we could not assess whether neighborhood characteristics affect individuals' risk of controlling hypertension or diabetes across time. Second, there is a slight temporal mismatch between the study period (2010-2011), HFAI data (collected in 2012), neighborhood SES measures (2010), and crime data (20062010); these data were the latest available data proximal to our study period. However, since neighborhood environment characteristics typically change slowly, we do not expect these discrepancies to affect the results significantly. Defining neighborhood SES at the census tract level may not correspond to actual geographic boundaries of a neighborhood as perceived by residents.

We excluded patients who were not engaged in care or did not have a recent clinical measure as these people lacked adequate information for comparison; however, these may be the most disadvantaged. However, our study criteria allow the study population to be inclusive of some patients who are engaged in care but are not optimally engaged in follow-up for hypertension or diabetes. We did not measure or adjust for treatment intensification and medication adherence due to the complexity of reliably measuring these factors in our EMR data; we acknowledge they are important for hypertension and diabetes control. Finally, there were an insufficient number of patients who were neither Black nor White in our sampling frame, and thus our results may not be generalizable to a more ethnically diverse group of patients.

Limited neighborhood variability among Black patients could have affected our ability to detect significant results. Historical factors such as racial and economic segregation ${ }^{34}$ create conditions in which a counterfactual comparison group of White patients residing in the same poor neighborhood environments as Blacks is limited, thereby restricting neighborhood variability of our predominantly Black patient population. However, while others may view neighborhood variability as a limitation, we recognize it captures a real-world phenomenon particularly for urban areas with large Black populations. Furthermore, because this study utilizes data from a clinical sample of patients who were somewhat engaged in primary care, study patients may not be representative of the overall population of residents in their respective Baltimore neighborhoods nor of the city overall.

Another major limitation in our study that may partially explain the differences in our findings compared to other prior studies is that we did not have specific information about the socioeconomic status (e.g., income level, employment history, or educational level) of the patients in this clinical sample. Specific information regarding individual socioeconomic status is not available within the EMR data that was extracted. Hence, without the ability to access and link data from other administrative sources or without data directly from patients regarding their socioeconomic status, we were unable to fully disentangle individual SES and neighborhood conditions in our analyses. Insurance coverage was the best proxy for individual SES available to us, but it is problematic because it was imperfect and incomplete. While we considered Medicaid insurance as a proxy for SES insofar as those eligible for Medicaid must be lower income, we were not able to fully distinguish between Medicare patients in the sample who were or were not dually eligible for Medicaid based on income status.

This study has several strengths, including being one of a few primary care-based studies to examine the association of multiple neighborhood environment characteristics on hypertension and diabetes control among patients seen within a 2year period. Another strength of this study is that we used detailed medical records data on clinical and individual factors among patients engaged in primary care, as most of the patients had clinical observations within 6 months of study baseline. Our study reflects citywide neighborhood composition with patients residing in 197 of the 200 census tracts in Baltimore despite not using a neighborhood-based sampling strategy. Finally, our inclusion criteria, patients receiving care within 2 years of study start date, were not overly restrictive, allowing us to capture patients across the continuum of care.

\section{CONCLUSIONS}

Our findings provide evidence extending existing literature on neighborhood environment and hypertension and diabetes control, which has focused primarily on community-based cohorts and disease prevalence. More recently, there are some studies examining clinical outcomes such as hypertension and diabetes control. This study does not find an independent association of neighborhood economic disadvantage, healthy food availability, or crime with hypertension and diabetes control. It does, however, find that, independent of these neighborhood factors, Black patients and patients on Medicaid or uninsured patients have lower odds of hypertension and diabetes control. It further highlights the inadequacies of current EMR data to fully describe the social context of patients, particularly insofar as individual SES is not routinely captured (e.g., via reported educational level, employment status, or income), which limits our ability to fully evaluate the independent association of neighborhood characteristics with clinical outcomes, since it is likely that some aspects of individual SES are not adequately measured. As such, this study supports the argument that health systems have an important role to play in identifying and characterizing patients' social context that is directly relevant to understanding and intervening to address neighborhood factors and social factors that affect chronic disease management. 
Acknowledgments: We would like to express our thanks to Kit Carson for her technical assistance related to the data acquisition.

Corresponding Author: Rachel L. J. Thornton, MD, PhD; Department of Pediatrics Johns Hopkins University School of Medicine, 5200 Eastern Avenue, Suite 4200, Baltimore, MD 21224, USA (e-mail: rjohns21@jhmi.edu).

Funding Information This work was supported by grants from the National Heart, Lung and Blood Institute (P5OHL0105187 [LAC, YJH], K24HL083113 [LAC], T32HL007180 [MM], and K23HL12150 [RLTT]). The content is solely the responsibility of the authors and does not necessarily represent the official views of the National Institutes of Health.

\section{Compliance with Ethical Standards:}

Conflict of Interest: The authors declare that they do not have a conflict of interest.

\section{REFERENCES}

1. Ettehad D, Emdin CA, Kiran A, et al. Blood pressure lowering for prevention of cardiovascular disease and death: a systematic review and meta-analysis. Lancet. 2016;387(10022):957-967.

2. Rapsomaniki E, Timmis A, George $\mathbf{J}$, et al. Blood pressure and incidence of twelve cardiovascular diseases: lifetime risks, healthy lifeyears lost, and age-specific associations in 1.25 million people. Lancet. 2014;383(9932): 1899-1911.

3. Benjamin EJ, Blaha MJ, Chiuve SE, et al. Heart Disease and Stroke Statistics-2017 Update: A Report From the American Heart Association. Circulation. 2017;135(10):e146-e603.

4. Carr LJ, Bartee RT, Dorozynski C, Broomfield JF, Smith ML, Smith DT. Internet-delivered behavior change program increases physical activity and improves cardiometabolic disease risk factors in sedentary adults: results of a randomized controlled trial. Prev Med. 2008;46(5):431-438.

5. Selvin E, Parrinello CM, Sacks DB, Coresh J. Trends in prevalence and control of diabetes in the United States, 1988-1994 and 1999-2010. Ann Intern Med. 2014;160(8):517-525.

6. Benoit SR, Fleming R, Philis-Tsimikas A, Ji M. Predictors of glycemic control among patients with Type 2 diabetes: a longitudinal study. BMC Public Health. 2005;5:36.

7. Wang $\mathbf{X}$, Auchincloss AH, Barber $\mathbf{S}$, et al. Neighborhood social environment as risk factors to health behavior among African Americans: The Jackson Heart Study. Health Place. 2017;45:199-207.

8. Yoon SS, Carroll MD, Fryar CD. Hypertension Prevalence and Control Among Adults: United States, 2011-2014. NCHS Data Brief. 2015(220):1-8.

9. Debnath S, Thameem F, Alves T, et al. Diabetic nephropathy among Mexican Americans. Clin Nephrol. 2012;77(4):332-344.

10. Tarver-Carr ME, Powe NR, Eberhardt MS, et al. Excess risk of chronic kidney disease among African-American versus white subjects in the United States: a population-based study of potential explanatory factors. $J$ Am Soc Nephrol. 2002; 13(9):2363-2370.

11. Diez Roux AV, Merkin SS, Arnett D, et al. Neighborhood of residence and incidence of coronary heart disease. N Engl J Med. 2001;345(2):99-106.

12. Leal C, Chaix B. The influence of geographic life environments on cardiometabolic risk factors: a systematic review, a methodological assessment and a research agenda. Obes Rev. 2011;12(3):217-230.

13. Bilal U, Auchincloss AH, Diez-Roux AV. Neighborhood Environments and Diabetes Risk and Control. Curr Diab Rep. 2018;18(9):62.

14. Buczynski AB, Freishtat H, Buzogany S. Mapping Baltimore City's Food Environment: 2015 Report. Johns Hopkins Center for a Livable Future;2015.

15. Franco M, Diez-Roux AV, Nettleton JA, et al. Availability of healthy foods and dietary patterns: the Multi-Ethnic Study of Atherosclerosis. Am J Clin Nutr. 2009;89(3):897-904.
16. Gucciardi E, Vahabi M, Norris N, Del Monte JP, Farnum C. The Intersection between Food Insecurity and Diabetes: A Review. Curr Nutr Rep. 2014;3(4):324-332.

17. Kelli HM, Kim JH, Samman Tahhan A, et al. Living in Food Deserts and Adverse Cardiovascular Outcomes in Patients With Cardiovascular Disease. J Am Heart Assoc. 2019;8(4):e010694.

18. Cooper LA, Marsteller JA, Noronha GJ, et al. A multi-level system quality improvement intervention to reduce racial disparities in hypertension care and control: study protocol. Implement Sci. 2013;8:60.

19. Diez-Roux AV, Kiefe CI, Jacobs DR, Jr., et al. Area characteristics and individual-level socioeconomic position indicators in three populationbased epidemiologic studies. Ann Epidemiol. 2001;11(6):395-405.

20. Diez Roux AV. Investigating neighborhood and area effects on health. Am J Public Health. 2001;91(11):1783-1789.

21. Jennings JM, Milam AJ, Greiner A, Furr-Holden CD, Curriero FC, Thornton RJ. Neighborhood alcohol outlets and the association with violent crime in one mid-Atlantic City: the implications for zoning policy. $J$ Urban Health. 2014;91(1):62-71.

22. Chobanian AV, Bakris GL, Black HR, et al. Seventh report of the Joint National Committee on Prevention, Detection, Evaluation, and Treatment of High Blood Pressure. Hypertension. 2003;42(6): 1206-1252.

23. Standards of Medical Care in Diabetes-2017: Summary of Revisions. Diabetes Care. 2017;40(Suppl 1):S4-S5.

24. Charlson ME, Pompei P, Ales KL, MacKenzie CR. A new method of classifying prognostic comorbidity in longitudinal studies: development and validation. J Chronic Dis. 1987;40(5):373-383.

25. Egan BM, Zhao Y, Axon RN. US trends in prevalence, awareness, treatment, and control of hypertension, 1988-2008. JAMA. 2010;303(20):2043-2050.

26. Tabaei BP, Rundle AG, Wu WY, et al. Associations of Residential Socioeconomic, Food, and Built Environments With Glycemic Control in Persons With Diabetes in New York City From 2007-2013. Am $J$ Epidemiol. 2018;187(4):736-745.

27. de Vries McClintock HF, Wiebe DJ, O'Donnell AJ, Morales KH, Small DS, Bogner HR. Neighborhood social environment and patterns of adherence to oral hypoglycemic agents among patients with type 2 diabetes mellitus. Fam Community Health. 2015;38(2):169-179.

28. Sims M, Diez Roux AV, Boykin S, et al. The socioeconomic gradient of diabetes prevalence, awareness, treatment, and control among African Americans in the Jackson Heart Study. Ann Epidemiol. 2011;21(12):892-898.

29. Weiner SJ, Schwartz A, Weaver F, et al. Contextual errors and failures in individualizing patient care: a multicenter study. Ann Intern Med. 2010; 153(2):69-75.

30. Morland K, Diez Roux AV, Wing S. Supermarkets, other food stores, and obesity: the atherosclerosis risk in communities study. Am J Prev Med. 2006;30(4):333-339.

31. Lovasi GS, Hutson MA, Guerra M, Neckerman KM. Built environments and obesity in disadvantaged populations. Epidemiol Rev. 2009;31:7-20.

32. Delamater A. Improving Patient Adherence. Clin Diabetes. 2006;24(2):71-77.

33. Krieger N. Embodiment: a conceptual glossary for epidemiology. $J$ Epidemiol Community Health. 2005;59(5):350-355.

34. Berube A, McDearman B. Good fortune, dire poverty, and inequality in Baltimore: An American story. In. Vol 2017. Brookings 2015.

35. Pietila A. Not in My Neighborhood: How Bigotry Shaped a Great American City.: Rowman \& Littlefield; 2012.

36. White $\mathbf{K}$, Haas JS, Williams DR. Elucidating the role of place in health care disparities: the example of racial/ethnic residential segregation. Health Serv Res. 2012;47(3 Pt 2):1278-1299.

37. Arnett DK, Blumenthal RS, Albert MA, et al. 2019 ACC/AHA Guideline on the Primary Prevention of Cardiovascular Disease: Executive Summary: A Report of the American College of Cardiology/American Heart Association Task Force on Clinical Practice Guidelines. J Am Coll Cardiol. 2019;74(10):1376-1414.

38. National Academies of Sciences, Engineering, and Medicine. Integrating Social Care into the Delivery of Health Care: Moving Upstream to Improve the Nation's Health. Washington: The National Academies Press 2019.

39. Hibbard $\mathbf{J H}$, Greene $\mathbf{J}$. What the evidence shows about patient activation: better health outcomes and care experiences; fewer data on costs. Health Aff (Millwood). 2013;32(2):207-214.

Publisher's Note Springer Nature remains neutral with regard to jurisdictional claims in published maps and institutional affiliations. 